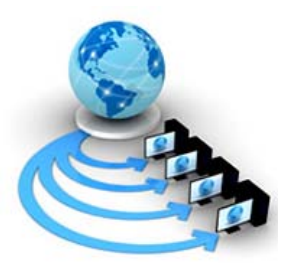

Volume 8, No. 9, November-December 2017

International Journal of Advanced Research in Computer Science

RESEARCH PAPER

Available Online at www.ijarcs.info

\title{
ROUGH TOPOLOGY ON APPROXIMATION SPACES
}

\author{
Sheeja. T. K \\ Department of Mathematics \\ T. M. Jacob Memorial Government College \\ Manimalakunnu, Kerala, India
}

\author{
Sunny Kuriakose. A \\ Professor and Dean \\ Federal Institute of Science and Technology \\ Angamaly, Kerala, India
}

\begin{abstract}
Though there are many studies on the topological properties of rough set approximations, only a few works have been done on the concept of rough topology. In this paper, a new definition of rough topology on an approximation space is proposed using the rough subsets of the extended approximation space. The basic concepts of a topological space are extended to the proposed rough topological space and the properties are investigated.
\end{abstract}

Keywords: lower approximation; upper approximation; rough Set; topology; rough topology.

\section{INTRODUCTION}

The theory of rough sets was formulated by Z. Pawlak in 1982 [18]. The mathematical framework of this potential theory has been enriched by the contribution of many researchers in various dimensions. An interesting and natural research area is the study of the interconnections between rough set theory and topology theory. The topological structure of rough sets forms an important base for information analysis and knowledge processing $[6,16,20,28]$.

There are mainly two streams of study connecting rough set theory and topology theory. The first one is regarding the topology induced by rough set approximations. In his seminal paper itself, Z. Pawlak [18] pointed out that the set of all equivalence classes in an approximation space formed a base for a topology on the set $U$ and the set of all equivalence classes of the rough equality relation formed a base for a topology on the power set of U. Both the topologies were found to be quasi discrete. Further studies on the topological properties of rough sets can be found in $[3,10,11,13,15,20,21,27,29]$. The second stream consists of the studies on the different types of approximation spaces induced by different topological spaces [1,6,12,23,24,25]. Some papers include results on both the streams [2,14]. Regarding information systems, T. Herawan [4,5] worked on the topology on an information system.

Though there are many studies on the relations between rough set approximations and topological spaces, only a few works are there on the concept of rough topology. $\mathrm{Q}$. Wu et al. [28] defined rough topology on a rough set by using a metric and then extended it to general topological space. M. L. Thivagar et al. [26] introduced the concept of rough topology which consists of the null set, the universal set, the lower approximation, upper approximation and the boundary region of a subset of U. B. P. Mathew and S. J. John [17] introduced the concept of rough topology on a rough set as a pair of topologies of exact subsets of the lower and upper approximations of the rough set under consideration. A follow up work was done by M. Ravindran and A. J. Divya [22] who studied the properties of compactness and connectedness and the separation axioms in rough topological spaces. But none of them regard rough topology as a rough subset of the power set.
In this paper, a new definition for rough topology on an approximation space is proposed using the rough subsets of the extended approximation space. The basic concepts of topology are extended to the proposed rough topology and the properties are investigated. This paper is organized as follows. In section 2, some preliminary concepts of rough set theory are recalled. In section 3 , the concept of rough topology on an approximation space is introduced. Section 4 deals with some of the basic concepts of rough topology and the conclusion is given in section 5 .

\section{Preliminaries}

In this section, some basic notions of rough set theory are recalled. Further details of rough set theory can be found in [19]. The basic concepts of topology theory are described in [9].

\section{A. Rough Set Theory}

The theory of rough sets was introduced by Z. Pawlak [18] in 1982. Consider the approximation space $(U, R)$, where $U$ is a non-empty set of objects and $R$ is an equivalence relation on $\mathrm{U}$. The equivalence classes of $\mathrm{R}$ are called elementary sets and sets which can be expressed as union of some equivalence classes are called composed sets.

The lower and upper approximations [19] of $A \subseteq U$, with respect to $\mathrm{R}$ are defined respectively as

$$
\begin{aligned}
& \underline{R}(A)=\left\{x \in U:[x]_{R} \subseteq A\right\} \\
& \bar{R}(A)=\left\{x \in U:[x]_{R} \cap A \neq \emptyset\right\}
\end{aligned}
$$

where, $[\mathrm{x}]_{\mathrm{R}}$ is the equivalence class of $\mathrm{R}$ containing $\mathrm{x}$. In other words, $\underline{R}(A)$ is the union of equivalence classes that are contained in $\mathrm{A}$ and $\bar{R}(A)$ is the union of equivalence classes that have non-empty intersection with A. Thus, both lower and upper approximations are composed sets on $(U, R)$.

The set $\underline{R}(A)$ is called the positive region, $U-\bar{R}(A)$ is called the negative region and the set $\bar{R}(A)-R(A)$ is called the boundary region. The properties of the rough set approximations are as follows:
1) $\underline{R}(\varnothing)=\bar{R}(\varnothing)=\varnothing$
2) $\underline{R}(X)=\bar{R}(X)=X$
3) $\underline{R}(A) \subseteq A \subseteq \bar{R}(A)$ 
4) $\underline{R}(\underline{R}(A))=\underline{R}(A)=\bar{R}(\underline{R}(A))$

5) $\bar{R}(\bar{R}(A))=\bar{R}(A)=\underline{R}(\bar{R}(A))$

6) $\underline{R}(A \cap B)=\underline{R}(A) \cap \underline{R}(B)$

7) $\overline{\bar{R}}(A \cap B) \subseteq \overline{\bar{R}}(A) \cap \overline{\bar{R}}(B)$

8) $\quad R(A \cup B) \supseteq \underline{R}(A) \cup R(B)$

9) $\overline{\bar{R}}(A \cup B)=\overline{\bar{R}}(A) \cup \overline{\bar{R}}(B)$

10) $\underline{R}(A)=\left(\bar{R}\left(A^{C}\right)\right)^{C}$

11) $\overline{\bar{R}}(A)=\left(\underline{R}(A)^{C}\right)^{C}$

12) $A \subseteq B \Rightarrow \underline{R}(A) \subseteq \underline{R}(B)$

13) $A \subseteq B \Rightarrow \overline{\bar{R}}(A) \subseteq \overline{\bar{R}}(B)$

14) $\underline{R}\left([x]_{R}\right)=[x]_{R}=\bar{R}\left([x]_{R}\right)$ for all $x \in X$

\section{B. Operations on Rough Sets}

The term 'rough set' has been used in two different viewpoints by Z. Pawlak. According to his original proposal, the equivalence classes of the rough equality relation were termed as rough sets [18]. Later, in [19], a subset $A \subseteq U$ with $\bar{R}(A) \neq \underline{R}(A)$ or $\operatorname{Bnd}(A) \neq \emptyset$ was called rough set. Rough sets may also be described using rough membership functions [19]. There is yet another approach to rough set theory proposed by T. B. Iwinski [7]. Throughout this paper, the word rough set refers to a pair $\left\langle X_{L}, X_{U}\right\rangle$, where $X_{L}$ and $X_{U}$ are subsets of $\mathrm{U}$ such that $X_{L}=\underline{R}(A)$ and $X_{U}=\bar{R}(A)$, for some $X \subseteq U$ [8]. For convenience, a rough set may be denoted by $\langle\underline{R}(A), \bar{R}(A)>$, where $A \subseteq U$.

The rough inclusion, rough union, rough intersection and rough complement operations of rough sets are defined in [8] as $\langle\underline{R}(A), \bar{R}(A)\rangle \subseteq\langle\underline{R}(B), \bar{R}(B)\rangle$

$$
\begin{aligned}
& \Leftrightarrow \underline{R}(A) \subseteq \underline{R}(B), \bar{R}(A) \subseteq \bar{R}(B) \\
&<\underline{R}(A), \bar{R}(A)>\cup<\underline{R}(B), \bar{R}(B)> \\
&=<\underline{R}(A) \cup \underline{R}(B), \bar{R}(A) \cup \bar{R}(B)> \\
&<\underline{R}(A), \bar{R}(A)>\cap<\underline{R}(B), \bar{R}(B)> \\
&=<\underline{R}(A) \cap \underline{R}(B), \bar{R}(A) \cap \bar{R}(B)> \\
&(<\underline{R}(A), \bar{R}(A)>)^{C}=<(\bar{R}(A))^{C},(\underline{R}(A))^{C}>
\end{aligned}
$$

respectively.

\section{B. Topology and Rough Sets}

Let $(U, R)$ be an approximation space. Then, the lower approximation operator satisfies the properties of an interior operator and hence it induces a topology $\mathcal{T}_{R}$ on $U$ [1]. A subset $A \in \mathcal{T}_{R}$ if and only if $\underline{R}(A)=A$. Hence, $\mathcal{T}_{R}$ consists of all composed sets on U. By properties (10) and (11), a set A will be closed iff $\bar{R}(A)=A$. Hence all open subsets are closed and $\mathcal{T}_{R}$ is the quasi discrete topology. The family of all equivalence classes form a basis for $\mathcal{T}_{R}$ [1].

Let $X=\left\langle X_{L}, X_{U}\right\rangle$ be a rough subset of the approximation space (U, R). Let $\mathcal{T}_{L}$ and $\mathcal{T}_{U}$ be any two topologies which contain only exact subsets of $X_{L}$ and $X_{U}$ respectively. Then the pair $\left\langle\mathcal{T}_{L}, \mathcal{T}_{U}\right\rangle$ is said to be a rough topology on U [17].

\section{ROUGH TOPOLOGY ON APPROXIMATION SPACES}

Let (U, R) be an approximation space, where $\mathrm{U}$ is a nonempty set of objects and $\mathrm{R}$ is an equivalence relation on $\mathrm{U}$. Consider the rough equality relation $\mathcal{R}$ on $\mathcal{P}(U)$, given by

$$
(A, B) \in \mathcal{R} \Leftrightarrow \underline{R}(A)=\underline{R}(B) \text { and } \bar{R}(A)=\bar{R}(B)
$$

Then, $\mathcal{R}$ is an equivalence relation on $\mathcal{P}(U)$ and the pair $(\mathcal{P}(U), \mathcal{R})$ is called the extended approximation space corresponding to $(\mathrm{U}, \mathrm{R})[1]$. Hence, we can extend the definition of rough set approximations to any subfamily $\mathcal{F} \subseteq \mathcal{P}(U)$.

\section{Definition 3.1:}

Let $(U, R)$ be an approximation space and $\mathcal{R}$ be the rough equality relation. Then, the $\mathcal{R}$-lower and $\mathcal{R}$-upper approximations of a sub family $\mathcal{F} \subseteq \mathcal{P}(U)$ are given by

$$
\begin{aligned}
& \mathcal{R}(\mathcal{F})=\left\{A \in \mathcal{P}(U):[A]_{\mathcal{R}} \subseteq \mathcal{F}\right\} \\
& \overline{\mathcal{R}}(\mathcal{F})=\left\{A \in \mathcal{P}(U):[A]_{\mathcal{R}} \cap \mathcal{F} \neq \emptyset\right\}
\end{aligned}
$$

respectively.

\section{Lemma 3.1:}

In the extended approximation space $(\mathcal{P}(U), \mathcal{R})$, $\forall x \in U,\left[[x]_{R}\right]_{\mathcal{R}}=\left\{[x]_{R}\right\}$.

Proof:

Since $\mathcal{R}$ is reflexive, $[x]_{R} \in\left[[x]_{R}\right]_{\mathcal{R}}$. Using property (14), $\underline{R}\left([x]_{R}\right)=[x]_{R}=\bar{R}\left([x]_{R}\right)$. Using eqn (7), if $A \in$ $\left[[x]_{R}\right]_{\mathcal{R}}$, then $\underline{R}(A)=\underline{R}\left([x]_{R}\right)=[x]_{R}=\bar{R}\left([x]_{R}\right)=\bar{R}(A)$. Thus $A=[x]_{R}$. Therefore, $\left[[x]_{R}\right]_{\mathcal{R}}=\left\{[x]_{R}\right\}$.

\section{Theorem 3.1:}

The $\mathcal{R}$-lower and $\mathcal{R}$-upper approximations of the quotient set $U / R$ is $U / R$ itself.

Proof:

We have, $\underline{\mathcal{R}}(U / R) \subseteq U / R$. If $A \in U / R$, then $A=[x]_{R}$ for some $x \in U$. So, $[A]_{\mathcal{R}}=\left[[x]_{R}\right]_{\mathcal{R}}=\left\{[x]_{R}\right\} \subseteq U / R$, using lemma 3.1. Hence, $A \in \underline{\mathcal{R}}(U / R)$. Thus, $\underline{\mathcal{R}}(U / R)=$ $U / R$. Similarly, $\overline{\mathcal{R}}(U / R)=U / R$.

\section{Lemma 3.2:}

If $\mathrm{A}$ is a composed set in $(U, R)$, then in the extended approximation space $(\mathcal{P}(U), \mathcal{R}),[A]_{\mathcal{R}}=\{A\}$.

Proof:

Obviously, $A \in[A]_{\mathcal{R}}$. Also, by the definition of $\mathcal{R}$, if $B \in[A]_{\mathcal{R}}$, then $\underline{R}(A)=\underline{R}(B)$ and $\bar{R}(A)=\bar{R}(B)$. Since A is a composed set, $\underline{R}(A)=A=\bar{R}(A)$. It follows that, $B=A$. Therefore, $[A]_{\mathcal{R}}=\{A\}$.

\section{Theorem 3.2:}

If $\mathcal{F} \subseteq \mathcal{P}(U)$ is a family of composed subsets of $\mathrm{U}$, then $\underline{\mathcal{R}}(\mathcal{F})=\mathcal{F}=\overline{\mathcal{R}}(\mathcal{F})$.

Proof:

By property (3), $\mathcal{R}(\mathcal{F}) \subseteq \mathcal{F}$. If $A \in \mathcal{F}$, then $\mathrm{A}$ is a composed set and by lemma 3.2, $[A]_{\mathcal{R}}=\{A\} \subseteq \mathcal{F}$. Hence, $A \in \underline{\mathcal{R}}(\mathcal{F})$ and so, $\mathcal{F} \subseteq \underline{\mathcal{R}}(\mathcal{F})$. Thus, $\underline{\mathcal{R}}(\mathcal{F})=\mathcal{F}$. Similarly, $\overline{\mathcal{R}}(\mathcal{F})=\mathcal{F}$.

\section{Corollary 3.1:}

$\underline{\mathcal{R}}\left(\tau_{R}\right)=\tau_{R}=\overline{\mathcal{R}}\left(\tau_{R}\right)$, where $\tau_{R}$ is the topology on $\mathrm{U}$ induced by $\mathrm{R}$.

Proof:

Being the topology on $\mathrm{U}$ induced by $\mathrm{R}, \tau_{R}$ consists of all composed subsets of U. Hence by theorem 3.2, $\underline{\mathcal{R}}\left(\tau_{R}\right)=$ $\tau_{R}=\overline{\mathcal{R}}\left(\tau_{R}\right)$.

\section{Definition 3.2:}

Let $\mathcal{T}$ be a subfamily of $\mathcal{P}(U)$. The pair $<\underline{\mathcal{R}}(\mathcal{T})$, $\overline{\mathcal{R}}(\mathcal{T})>$ is called an $\mathcal{R}$-rough topology on U, if both $\underline{\mathcal{R}}(\mathcal{T})$ and $\overline{\mathcal{R}}(\mathcal{T})$ are topologies on $\mathrm{U}$. The set $\mathrm{U}$ together with the $\mathcal{R}$-rough topology is called an $\mathcal{R}$-rough topological space. 


\section{Definition 3.3:}

Let $\mathcal{T}$ be a subfamily of $\mathcal{P}(U)$. The pair $<\underline{\mathcal{R}}(\mathcal{T})$, $\overline{\mathcal{R}}(\mathcal{T})>$ is called a lower $\mathcal{R}$-rough topology on $\mathrm{U}$, if $\underline{\mathcal{R}}(\mathcal{T})$ is a topology on $\mathrm{U}$.

\section{Definition 3.4:}

Let $\mathcal{T}$ be a subfamily of $\mathcal{P}(U)$. The pair $<\underline{\mathcal{R}}(\mathcal{T})$, $\overline{\mathcal{R}}(\mathcal{T})>$ is called an upper $\mathcal{R}$-rough topology on $U$, if $\overline{\mathcal{R}}(\mathcal{T})$ is a topology on $U$.

\section{Definition 3.5:}

Let $\langle\underline{\mathcal{R}}(\mathcal{T}), \overline{\mathcal{R}}(\mathcal{T})>$ be an $\mathcal{R}$-rough topology on $U$. The rough set $\langle\underline{R}(A), \bar{R}(A)\rangle$ is called lower $\mathcal{R}$-rough open if $\underline{R}(A)$ is an open set in $\underline{\mathcal{R}}(\mathcal{T})$, upper $\mathcal{R}$-rough open if $\bar{R}(A)$ is an open set in $\overline{\mathcal{R}}(\mathcal{T})$ and $\mathcal{R}$-rough open if it is both lower $\mathcal{R}$-rough open and upper $\mathcal{R}$-rough open.

\section{Example 3.1:}

Let $U=\{a, b, c, d\}$ and consider the equivalence relation given by $U / R=\{\{a, b\},\{c\},\{d\}\}$. The induced equivalence relation on $\mathcal{P}(U)$ is given by $\mathcal{P}(U) / \mathcal{R}=$ $\{\varnothing,\{U\},\{\{d\}\},\{\{c\}\},\{\{a\},\{b\}\},\{\{a, b\}\},\{\{c, d\}\},\{\{a, c\},\{b, c\}\}$, $\{\{a, d\},\{b, d\}\},\{\{a, b, c\}\},\{\{a, b, d\}\},\{\{a, c, d\},\{b, c, d\}\}\}$.

Let $\mathcal{T}_{1}=\{\varnothing, U,\{a\},\{c\}\}$. Then, $\underline{\mathcal{R}}\left(\mathcal{T}_{1}\right)=\{\varnothing, U,\{c\}\}$ is a topology on $\mathrm{U}$ and $\overline{\mathcal{R}}\left(\mathcal{T}_{1}\right)=\{\varnothing, U,\{a\},\{b\},\{c\}\}$ is not a topology on U. Thus, $<\underline{\mathcal{R}}\left(\mathcal{T}_{1}\right), \overline{\mathcal{R}}\left(\mathcal{J}_{1}\right)>$ is a lower $\mathcal{R}$-rough topology on $\mathrm{U}$, which is neither an upper $\mathcal{R}$-rough topology nor an $\mathcal{R}$-rough topology.

\section{Example 3.2:}

Let $\mathcal{T}_{2}=\{\varnothing, U,\{a, c, d\},\{c, d\}$ in the previous example. Clearly, both $\overline{\mathcal{R}}\left(\mathcal{T}_{2}\right)=\{\{\varnothing, U,\{a, c, d\},\{b, c, d\},\{c, d\}\}$ and $\underline{\mathcal{R}}\left(\mathcal{T}_{2}\right)=\{\varnothing, U,\{c, d\}\}$ are topologies on U. Therefore, $<\underline{\mathcal{R}}\left(\mathcal{T}_{1}\right), \overline{\mathcal{R}}\left(\mathcal{T}_{1}\right)>$ is a lower $\mathcal{R}$-rough topology and an upper $\mathcal{R}$-rough topology. Hence, it is an $\mathcal{R}$-rough topology on $\mathrm{U}$.

The pair $<\{c, d\},\{a, b, c, d\}>$ is an $\mathcal{R}$-rough open set.

\section{Proposition 3.1:}

If $\langle\underline{R}(A), \bar{R}(A)>$ is a lower $\mathcal{R}$-rough open set, then $<\underline{R}(A), \underline{R}(A)>$ is an $\mathcal{R}$-rough open set.

Proof:

Since $\langle\underline{R}(A), \bar{R}(A)>$ is a lower $\mathcal{R}$-rough open set, $\underline{R}(A) \in \underline{\mathcal{R}}(\mathcal{T})$. Also, $\underline{\mathcal{R}}(\mathcal{T}) \subseteq \overline{\mathcal{R}}(\mathcal{T})$. Hence, $\underline{R}(A) \in \overline{\mathcal{R}}(\mathcal{T})$. Therefore, $<\underline{R}(A), \underline{R}(A)>$ is an $\mathcal{R}$-rough open set.

\section{Theorem 3.3:}

Let $<\underline{\mathcal{R}}(\mathcal{T}), \overline{\mathcal{R}}(\mathcal{T})>$ be an $\mathcal{R}$-rough topology on $\mathrm{U}$. Then, the family of all $\mathcal{R}$-rough open sets on $\mathrm{U}$ is a topology on $\mathcal{P}(U) \times \mathcal{P}(U)$.

Proof:

Let $\mathfrak{I}_{\mathcal{T}}$ denote the family of all $\mathcal{R}$-rough open sets on $U$. Since $<\underline{\mathcal{R}}(\mathcal{T}), \overline{\mathcal{R}}(\mathcal{T})>$ is an $\mathcal{R}$-rough topology on U, both $\underline{\mathcal{R}}(\mathcal{T})$ and $\overline{\mathcal{R}}(\mathcal{T})$ are topologies on U. Hence, $\emptyset \in \underline{\mathcal{R}}(\mathcal{T})$ and $\bar{\emptyset} \in \overline{\mathcal{R}}(\mathcal{T})$. So, $\left\langle\emptyset, \emptyset>\in \mathfrak{I}_{\mathcal{T}}\right.$. Similarly, $\langle U, U\rangle \in \mathfrak{I}_{\mathcal{T}}$ as $U \in \underline{\mathcal{R}}(\mathcal{T})$ and $U \in \overline{\mathcal{R}}(\mathcal{T})$.

If $<\underline{R}(A), \bar{R}(A)>\in \mathfrak{T}_{\mathcal{T}}$ and $<\underline{R}(B), \bar{R}(B)>\in \mathfrak{I}_{\mathcal{T}}$, then, $\underline{R}(A) \cap \underline{R}(B) \in \underline{\mathcal{R}}(\mathcal{T})$ and $\bar{R}(A) \cap \bar{R}(B) \in \overline{\mathcal{R}}(\mathcal{T})$. So, $<\underline{R}(A), \bar{R}(A)>\cap<\underline{R}(B), \bar{R}(B)>=<\underline{R}(A) \cap \underline{R}(B)$,
$\bar{R}(A) \cap \bar{R}(B)>\in \mathfrak{I}_{\mathcal{T}}$. Also, if $<\underline{R}\left(A_{i}\right), \bar{R}\left(A_{i}\right)>\in \mathfrak{I}_{\mathcal{T}}$ for $i \in \Lambda$, then, $\cup \underline{R}\left(A_{i}\right) \in \underline{\mathcal{R}}(\mathcal{T})$ and $\cup \bar{R}\left(A_{i}\right) \in \overline{\mathcal{R}}(\mathcal{T})$. Thus, $\bigcup\left\{<\underline{R}\left(A_{i}\right), \bar{R}\left(A_{i}\right)>\right\}=<\cup \underline{R}\left(A_{i}\right), \cup \bar{R}\left(A_{i}\right)>\in \mathfrak{I}_{\mathcal{T}}$.

Therefore, $\mathfrak{I}_{\mathcal{T}}$ is a topology on $\mathcal{P}(U) \times \mathcal{P}(U)$. Thus, the finite rough intersection of $\mathcal{R}$-rough open sets is $\mathcal{R}$ rough open and arbitrary rough union of $\mathcal{R}$-rough open sets is $\mathcal{R}$ rough open.

\section{Definition 3.6:}

Let $<\underline{\mathcal{R}}\left(\mathcal{T}_{1}\right), \overline{\mathcal{R}}\left(\mathcal{T}_{1}\right)>$ and $<\underline{\mathcal{R}}\left(\mathcal{T}_{2}\right), \overline{\mathcal{R}}\left(\mathcal{T}_{2}\right)>$ be two $\mathcal{R}$ rough topologies on $\mathrm{U}$. Then, $\left\langle\underline{\mathcal{R}}\left(\mathcal{T}_{1}\right), \overline{\mathcal{R}}\left(\mathcal{T}_{1}\right)>\right.$ is said to be stronger than $<\underline{\mathcal{R}}\left(\mathcal{T}_{2}\right), \overline{\mathcal{R}}\left(\mathcal{T}_{2}\right)>$ iff $\mathfrak{I}_{\mathcal{T}_{1}} \supseteq \mathfrak{I}_{\mathcal{T}_{2}}$.

\section{Definition 3.7:}

Let $<\underline{\mathcal{R}}\left(\mathcal{T}_{1}\right), \overline{\mathcal{R}}\left(\mathcal{T}_{1}\right)>$ and $<\underline{\mathcal{R}}\left(\mathcal{T}_{2}\right), \overline{\mathcal{R}}\left(\mathcal{T}_{2}\right)>$ be two $\mathcal{R}$ -rough topologies on U. Then, $<\underline{\mathcal{R}}\left(\mathcal{T}_{1}\right), \overline{\mathcal{R}}\left(\mathcal{T}_{1}\right)>$ is said to be equivalent to $<\underline{\mathcal{R}}\left(\mathcal{T}_{2}\right), \overline{\mathcal{R}}\left(\mathcal{T}_{2}\right)>$ iff $\mathfrak{I}_{\mathcal{T}_{1}}=\mathfrak{I}_{\mathcal{T}_{2}}$.

The following theorem shows that the approximations of the topology induced by $R$ constitute an $\mathcal{R}$-rough topology on $U$.

\section{Theorem 3.4:}

The pair $<\underline{\mathcal{R}}\left(\tau_{R}\right), \overline{\mathcal{R}}\left(\tau_{R}\right)>$ is an $\mathcal{R}$-rough topology on $\mathrm{U}$, where $\tau_{R}$ is the topology on $U$ induced by $R$. Proof:

Since $\mathrm{R}$ is an equivalence relation on $\mathrm{U}, \tau_{R}$ consists of all composed subsets of U. Hence, $\underline{\mathcal{R}}\left(\tau_{R}\right)=\tau_{R}=\overline{\mathcal{R}}\left(\tau_{R}\right)$, by theorem 3.2. Therefore, both $\underline{\mathcal{R}}\left(\tau_{R}\right)$ and $\overline{\mathcal{R}}\left(\tau_{R}\right)$ are topologies on $U$. Thus, $<\underline{\mathcal{R}}\left(\tau_{R}\right), \overline{\overline{\mathcal{R}}}\left(\tau_{R}\right)>$ is an $\mathcal{R}$-rough topology on $U$.

\section{Theorem 3.5:}

Every rough subset of $U$ is $\mathcal{R}$-rough open in the $\mathcal{R}$ rough topology $<\underline{\mathcal{R}}\left(\tau_{R}\right), \overline{\mathcal{R}}\left(\tau_{R}\right)>$.

Proof:

Let $<\underline{R}(A), \bar{R}(A)>$ be a rough set on U. Since both $\underline{R}(A)$ and $\overline{\bar{R}}(A)$ are union of equivalence classes, $\underline{R}(A) \in \tau_{R}$ and $\bar{R}(A) \in \tau_{R}$. Also, $\underline{\mathcal{R}}\left(\tau_{R}\right)=\tau_{R}=\overline{\mathcal{R}}\left(\tau_{R}\right)$. So, $\underline{R}(A) \in$ $\underline{\mathcal{R}}\left(\tau_{R}\right)$ and $\bar{R}(A) \in \overline{\mathcal{R}}\left(\tau_{R}\right)$. Therefore, $\langle\underline{R}(A), \bar{R}(\bar{A})\rangle$ is $\overline{\mathcal{R}}$-rough open.

Next, we present the definition of discrete $\mathcal{R}$-rough topology on $U$.

\section{Definition 3.8:}

Any $\mathcal{R}$-rough topology on $\mathrm{U}$ which is equivalent to $<\underline{\mathcal{R}}\left(\tau_{R}\right), \overline{\mathcal{R}}\left(\tau_{R}\right)>$ is called a discrete $\mathcal{R}$-rough topology.

\section{Theorem 3.6:}

The pair $<\underline{\mathcal{R}}\left(\tau_{D}\right), \overline{\mathcal{R}}\left(\tau_{D}\right)>$ is an $\mathcal{R}$-rough topology on $\mathrm{U}$, where $\tau_{D}$ is the discrete topology on $\mathrm{U}$. Proof:

The discrete topology on $U$ consists of all subsets of $U$. Hence, $\tau_{D}=\mathcal{P}(U)$. Since, $\underline{\mathcal{R}}(\mathcal{P}(U))=\mathcal{P}(U)=\overline{\mathcal{R}}(\mathcal{P}(U))$, we get, $\underline{\mathcal{R}}\left(\tau_{D}\right)=\tau_{D}=\overline{\mathcal{R}}\left(\tau_{D}\right)$. Therefore, both $\underline{\mathcal{R}}\left(\tau_{D}\right)$ and are topologies on U. Thus, $\left\langle\underline{\mathcal{R}}\left(\tau_{D}\right), \overline{\mathcal{R}}\left(\tau_{D}\right)>\right.$ is an $\mathcal{R}$ rough topology on $\mathrm{U}$.

\section{Theorem 3.7:}

The pair $<\underline{\mathcal{R}}\left(\tau_{D}\right), \overline{\mathcal{R}}\left(\tau_{D}\right)>$ is a discrete $\mathcal{R}$-rough topology on $\mathrm{U}$.

Proof: 
If $<R(A), \bar{R}(A)>$ is an $\mathcal{R}$-rough open set with respect to $\tau_{D}$, then, using theorem $3.5,<\underline{R}(A), \bar{R}(A)>$ is $\mathcal{R}$-rough open with respect to $\tau_{R}$. Therefore, $\mathfrak{I}_{\tau_{D}} \subseteq \mathfrak{I}_{\tau_{R}}$. Conversely, if $<\underline{R}(B), \bar{R}(B)>\in \mathfrak{I}_{\tau_{R}}$, then, $\underline{R}(B) \in \underline{\mathcal{R}}\left(\tau_{D}\right)$ and $\bar{R}(B) \in \overline{\mathcal{R}}\left(\tau_{D}\right)$, because $\underline{\mathcal{R}}\left(\tau_{D}\right)=\tau_{D}=\overline{\mathcal{R}}\left(\tau_{D}\right)$. So, $<\underline{R}(B), \bar{R}(B)>\in \mathfrak{I}_{\tau_{D}}$. Therefore, , $\mathfrak{I}_{\tau_{R}} \subseteq \mathfrak{I}_{\tau_{D}}$. Thus, $\mathfrak{I}_{\tau_{D}}=\mathfrak{I}_{\tau_{R}}$. Hence, $<\underline{\mathcal{R}}\left(\tau_{D}\right), \overline{\mathcal{R}}\left(\tau_{D}\right)>$ is equivalent to $<\underline{\mathcal{R}}\left(\tau_{R}\right), \overline{\mathcal{R}}\left(\tau_{R}\right)>$. Therefore, $\left\langle\underline{\mathcal{R}}\left(\tau_{D}\right), \overline{\mathcal{R}}\left(\tau_{D}\right)>\right.$ is a discrete $\mathcal{R}$-rough topology on $U$.

\section{Theorem 3.8:}

The pair $<\underline{\mathcal{R}}\left(\tau_{I}\right), \overline{\mathcal{R}}\left(\tau_{I}\right)>$ is an $\mathcal{R}$-rough topology on $U$, where $\tau_{I}$ is the indiscrete topology on $U$. Proof:

The indiscrete topology consists of only $\emptyset$ and $U$, which are composed sets on $U$. Hence, $\underline{\mathcal{R}}\left(\tau_{I}\right)=\tau_{I}=\overline{\mathcal{R}}\left(\tau_{I}\right)$. Therefore, $\underline{\mathcal{R}}\left(\tau_{I}\right)$ and $\overline{\mathcal{R}}\left(\tau_{I}\right)$ are topologies on $U$. Thus, $<\underline{\mathcal{R}}\left(\tau_{I}\right), \overline{\mathcal{R}}\left(\tau_{I}\right)>$ is an $\mathcal{R}$-rough topology on $U$.

\section{Definition 3.9:}

Any $\mathcal{R}$-rough topology on $U$ which is equivalent to $<\underline{\mathcal{R}}\left(\tau_{I}\right), \overline{\mathcal{R}}\left(\tau_{I}\right)>$ is called an indiscrete $\mathcal{R}$-rough topology.

\section{Remark 3.1:}

The rough sets $\langle\emptyset, \emptyset\rangle$ and $\langle U, U\rangle$ are $\mathcal{R}$-rough open sets in the indiscrete $\mathcal{R}$-rough topology on $\mathrm{U}$. However, if $\langle\emptyset, U\rangle$ and $\langle U, \emptyset\rangle$ are rough sets, then they are also $\mathcal{R}$-rough open sets in the indiscrete $\mathcal{R}$-rough topology on $\mathrm{U}$.

\section{ROUGH CLOSED SETS, ROUGH CLOSURE AND ROUGH INTERIOR}

In this section, some basic concepts of a topological space such as rough closed set, rough interior and rough closure are extended to the proposed rough topological space and the properties are investigated.

\section{Definition 4.1:}

Let $<\underline{\mathcal{R}}(\mathcal{T}), \overline{\mathcal{R}}(\mathcal{T})>$ be an $\mathcal{R}$-rough topology on $\mathrm{U}$. Then the rough set $\langle\underline{R}(A), \bar{R}(A)\rangle$ is respectively called lower $\mathcal{R}$-rough closed, upper $\mathcal{R}$-rough closed and $\mathcal{R}$-rough closed if $\left\langle\underline{R}\left(A^{C}\right), \bar{R}\left(A^{C}\right)\right\rangle$ is lower $\mathcal{R}$-rough open, upper $\mathcal{R}$-rough open or $\mathcal{R}$-rough open.

\section{Theorem 4.1:}

A rough set $<\underline{R}(A), \bar{R}(A)>$ is $\mathcal{R}$-rough closed iff its rough complement is $\mathcal{R}$-rough open.

Proof:

We have, $<\underline{R}(A), \bar{R}(A)>$ is $\mathcal{R}$-rough closed

$$
\begin{aligned}
& \Leftrightarrow<\underline{R}\left(A^{C}\right), \bar{R}\left(A^{C}\right)>\text { is } \mathcal{R} \text {-rough open } \\
& \Leftrightarrow<(\bar{R}(A))^{C},(\underline{R}(A))^{C},>\text { is } \mathcal{R} \text {-rough open } \\
& \Leftrightarrow(<\underline{R}(A), \bar{R}(A)>)^{C} \text { is } \mathcal{R} \text {-rough open }
\end{aligned}
$$

\section{Corollary 4.1:}

The rough sets $\langle\emptyset, \emptyset\rangle$ and $\langle U, U\rangle$ are $\mathcal{R}$-rough closed in any $\mathcal{R}$-rough topology on $\mathrm{U}$.

Proof:

As $(<\emptyset, \emptyset\rangle)^{C}=\langle U, U\rangle$ and $\left.(<U, U\rangle\right)^{C}=<$ $\emptyset, \emptyset>$ are $\mathcal{R}$-rough open sets with respect to any $\mathcal{R}$-rough topology on $\mathrm{U},\langle\emptyset, \emptyset\rangle$ and $\langle U, U\rangle$ are $\mathcal{R}$-rough closed in any $\mathcal{R}$-rough topology on $\mathrm{U}$.

\section{Theorem 4.2:}

Let $<\underline{\mathcal{R}}(\mathcal{T}), \overline{\mathcal{R}}(\mathcal{T})>$ be an $\mathcal{R}$-rough topology on $\mathrm{U}$ and $\mathcal{F}_{\mathcal{T}}$ denote the family of all $\mathcal{R}$-rough closed subsets of $\mathrm{U}$. Then,

1. $\left\langle\emptyset, \emptyset>\in \mathcal{F}_{\mathcal{T}},\left\langle U, U>\in \mathcal{F}_{\mathcal{T}}\right.\right.$

2. $\mathcal{F}_{\mathcal{T}}$ is closed under finite rough union.

3. $\mathcal{F}_{\mathcal{T}}$ is closed under arbitrary rough intersection Proof:

From theorem 4.1, $<\underline{R}(A), \bar{R}(A)>$ is $\mathcal{R}$-rough closed iff $(<\underline{R}(A), \bar{R}(A)>)^{C}$ is $\bar{R}$-rough open.

1. By corollary $4.1,<\emptyset, \emptyset>\in \mathcal{F}_{\mathcal{T}},\left\langle U, U>\in \mathcal{F}_{\mathcal{T}}\right.$.

2. $<\underline{R}(A), \bar{R}(A)>\in \mathcal{F}_{\mathcal{T}}, \quad<\underline{R}(B), \bar{R}(B)>\in \mathcal{F}_{\mathcal{T}} \Rightarrow$ $(<\underline{R}(A), \bar{R}(A)>)^{C} \in \mathfrak{I}_{\mathcal{T}},(<\underline{R}(B), \bar{R}(B)>)^{C} \in \mathfrak{I}_{\mathcal{T}}$ $\Rightarrow<\underline{R}(A), \bar{R}(A)>^{C} \cap<\underline{R}(B), \bar{R}(B)>^{C} \in \mathfrak{I}_{\mathcal{T}}$ $\Rightarrow(<\underline{R}(A), \bar{R}(A)>\cup<\underline{R}(B), \bar{R}(B)>)^{C} \in \mathfrak{I}_{\mathcal{T}}$ $\Rightarrow<\underline{R}(A), \bar{R}(A)>\cup<\underline{R}(B), \bar{R}(B)>\in \mathcal{F}_{\mathcal{T}}$

Thus $\mathcal{F}_{\mathcal{T}}$ is closed under finite rough union.

3. $<\underline{R}\left(A_{i}\right), \bar{R}\left(A_{i}\right)>\in \mathcal{F}_{\mathcal{T}}$ for $i \in \Lambda$

$\Rightarrow<\underline{R}\left(A_{i}\right), \bar{R}\left(A_{i}\right)>^{C} \in \mathfrak{I}_{\mathcal{T}}$ for $i \in \Lambda$

$\Rightarrow \mathrm{U}_{i \in \Lambda}<\underline{R}\left(A_{i}\right), \bar{R}\left(A_{i}\right)>^{C} \in \mathfrak{I}_{\mathcal{T}}$

$\Rightarrow\left(\bigcap_{i \in \Lambda}<\underline{R}\left(A_{i}\right), \bar{R}\left(A_{i}\right)>\right)^{C} \in \mathfrak{I}_{\mathcal{T}}$

$\Rightarrow \bigcap_{i \in \Lambda}<\underline{R}\left(A_{i}\right), \bar{R}\left(A_{i}\right)>\in \mathcal{F}_{\mathcal{T}}$

Thus $\mathcal{F}_{\mathcal{T}}$ is closed under arbitrary rough intersection.

\section{Definition 4.2:}

Let $<\underline{\mathcal{R}}(\mathcal{T}), \overline{\mathcal{R}}(\mathcal{T})>$ be an $\mathcal{R}$-rough topology on U. Then the $\mathcal{R}$-rough interior denoted by $\operatorname{Rint}(<\underline{R}(A), \bar{R}(A)>)$ of a rough set $<\underline{R}(A), \bar{R}(A)>$ is defined as the union of all $\mathcal{R}$ -rough open sets contained in $\langle\underline{R}(A), \bar{R}(A)>$.

\section{Definition 4.3:}

Let $<\underline{\mathcal{R}}(\mathcal{T}), \overline{\mathcal{R}}(\mathcal{T})>$ be an $\mathcal{R}$-rough topology on U. Then the $\mathcal{R}$-rough closure denoted by $\operatorname{Rcl}(<\underline{R}(A), \bar{R}(A)>)$ of a rough set $\langle\underline{R}(A), \bar{R}(A)>$ is defined as the intersection of all $\mathcal{R}$-rough closed sets containing $<\underline{R}(A), \bar{R}(A)>$.

\section{Remark 4.1:}

From the definition, $\operatorname{Rint}(<\underline{R}(A), \bar{R}(A)>)$ is the largest $\mathcal{R}$-rough open set contained in $\langle\underline{R}(A), \bar{R}(A)\rangle$ and $\mathcal{R} c l(<\underline{R}(A), \bar{R}(A)>)$ is the smallest $\overline{\mathcal{R}}$-rough closed set containing $<\underline{R}(A), \bar{R}(A)>$.

\section{Theorem 4.3:}

Let $<\underline{\mathcal{R}}(\mathcal{T}), \overline{\mathcal{R}}(\mathcal{T})>$ be an $\mathcal{R}$-rough topology on $\mathrm{U}$ and $<\underline{R}(A), \bar{R}(A)>$ be a rough subset of $\mathrm{U}$. Then,

1. $<\underline{R}(A), \bar{R}(A)>\in \mathfrak{I}_{\mathcal{T}}$ iff

$$
\operatorname{Rint}(<\underline{R}(A), \bar{R}(A)>)=\langle\underline{R}(A), \bar{R}(A)>
$$

2. Rint $\langle\emptyset, \emptyset\rangle=\langle\emptyset, \emptyset\rangle$, Rint $\langle U, \bar{U}\rangle=\langle U, U\rangle$

3. $\operatorname{Rint}(\mathcal{R i n t}(<\underline{R}(A), \bar{R}(A)>))$

$$
=\operatorname{Rint}(<\underline{R}(A), \bar{R}(A)>)
$$

4. $\operatorname{Rint}(<\underline{R}(A), \bar{R}(A)>\cap<\underline{R}(B), \bar{R}(B)>)=$ $\operatorname{Rint}(<\underline{R}(A), \bar{R}(A)>) \cap \operatorname{Rint}(<\underline{R}(B), \bar{R}(B)>)$ Proof:

1. $<\underline{R}(A), \bar{R}(A)>\in \mathfrak{I}_{\mathcal{T}} \Leftrightarrow$ the largest $\mathcal{R}$-open set contained in $\langle\underline{R}(A), \bar{R}(A)>$ is $\langle\underline{R}(A), \bar{R}(A)>$ $\Leftrightarrow \operatorname{Rint}(<\underline{R}(A), \bar{R}(A)>)=<\underline{R}(A), \bar{R}(A)>$. 
2. Both $\langle\emptyset, \emptyset\rangle$ and $\langle U, U\rangle$ are $\mathcal{R}$-rough open sets. Hence, from (1) we get, Rint $\langle\emptyset, \emptyset\rangle=\langle\emptyset, \emptyset\rangle$ and $\operatorname{Rint}\langle U, U\rangle=\langle U, U\rangle$.

3. $\operatorname{Rint}(<\underline{R}(A), \bar{R}(A)>)$ is an $\mathcal{R}$-rough open set. So, $\operatorname{Rint}(\mathcal{R i n t}(<\underline{R}(A), \bar{R}(A)>))=$

$$
\operatorname{Rint}(<\underline{R}(A), \bar{R}(A)>)
$$

4. $\operatorname{Rint}(<\underline{R}(A), \bar{R}(A)>\bar{\cap}<\underline{R}(B), \bar{R}(B)>)=$ $\operatorname{Rint}(<\underline{R}(A) \cap \underline{R}(B), \bar{R}(A) \cap \bar{R}(B)>)$. Also, $<\underline{R}(X), \bar{R}(X)>\subseteq<\underline{R}(A) \cap \underline{R}(B), \bar{R}(A) \cap \bar{R}(B)>$ $\Rightarrow \underline{R}(X) \subseteq \underline{R}(A) \cap \underline{R}(B), \bar{R}(X) \subseteq \bar{R}(A) \cap \bar{R}(B)$ $\Rightarrow \underline{R}(X) \subseteq \underline{R}(A), \underline{R}(X) \subseteq \underline{R}(B)$ and $\overline{\bar{R}}(X) \subseteq \bar{R}(A), \bar{R}(X) \subseteq \bar{R}(B)$

$\Rightarrow \underline{R}(X) \subseteq \underline{R}(A), \bar{R}(X) \subseteq \bar{R}(A)$ and $\underline{R}(X) \subseteq \underline{R}(B), \bar{R}(X) \subseteq \bar{R}(B)$

$\Rightarrow<\underline{R}(X), \bar{R}(X)>\subseteq<\underline{R}(A), \bar{R}(A)>$ and $<\underline{R}(X), \bar{R}(X)>\subseteq<\underline{R}(B), \bar{R}(B)>$

Thus, $\operatorname{Rint}(<\underline{R}(A), \bar{R}(A)>\cap<\underline{R}(B), \bar{R}(B)>)$ $\subseteq \mathcal{R} \operatorname{Rint}(<\underline{R}(A), \bar{R}(A)>) \cap \operatorname{Rint}(<\underline{R}(B), \bar{R}(B)>)$. $\mathcal{R i n t}(<\underline{R}(A), \bar{R}(A)>) \cap \mathcal{R i n t}(<\underline{R}(B), \bar{R}(B)>)$ is an $\mathcal{R}$-rough open set contained in $\langle\underline{R}(A), \bar{R}(A)\rangle$ and $<\underline{R}(B), \bar{R}(B)>$. Therefore,

$\operatorname{Rint}(<\underline{R}(A), \bar{R}(A)>) \cap \mathcal{R i n t}(<\underline{R}(B), \bar{R}(B)>)$ $\subseteq \mathcal{R} \operatorname{int}(<\underline{R}(A), \bar{R}(A)>\cap<\underline{R}(B), \bar{R}(B)>)$. Hence, $\mathcal{R i n t}(<\underline{R}(A), \bar{R}(A)>) \cap \mathcal{R i n t}(<\underline{R}(B), \bar{R}(B)>)$ $=\operatorname{Rint}(<\underline{R}(A), \bar{R}(A)>\cap<\underline{R}(B), \bar{R}(B)>)$.

\section{Theorem 4.4:}

Let $<\underline{\mathcal{R}}(\mathcal{T}), \overline{\mathcal{R}}(\mathcal{T})>$ be an $\mathcal{R}$-rough topology on $\mathrm{U}$ and $<\underline{R}(A), \bar{R}(A)>$ be a rough subset of $\mathrm{U}$. Then,

1. $<\underline{R}(A), \bar{R}(A)>\in \mathcal{F}_{\mathcal{T}}$ iff $\operatorname{Rcl}(<\underline{R}(A), \bar{R}(A)>)=\langle\underline{R}(A), \bar{R}(A)>$

2. $\mathcal{R} c l<\emptyset, \emptyset\rangle=\langle\emptyset, \emptyset\rangle, \mathcal{R} c l\langle U, U\rangle=\langle U, U\rangle$

3. $\mathcal{R c l}(\mathcal{R} c l(<\underline{R}(A), \bar{R}(A)>))$

$$
=\mathcal{R} c l(<\underline{R}(A), \bar{R}(A)>)
$$

4. $\operatorname{Rcl}(<\underline{R}(A), \bar{R}(A)>\cup<\underline{R}(B), \bar{R}(B)>)=$ $\mathcal{R} c l(<\underline{R}(A), \bar{R}(A)>) \cup \operatorname{Rcl}(<\underline{R}(B), \bar{R}(B)>)$

Proof:

1. $<\underline{R}(A), \bar{R}(A)>\in \mathcal{F}_{\mathcal{T}} \Leftrightarrow$ the smallest $\mathcal{R}$-closed set containing $\langle\underline{R}(A), \bar{R}(A)\rangle$ is $\langle\underline{R}(A), \bar{R}(A)\rangle$ itself $\Leftrightarrow \mathcal{R} c l(\langle\underline{R}(A), \bar{R}(A)\rangle)=\langle\underline{R}(A), \bar{R}(A)\rangle$.

2. Both $\langle\emptyset, \emptyset\rangle$ and $\langle U, U\rangle$ are $\mathcal{R}$-rough closed sets. Hence, using (1), we get $\mathcal{R} c l\langle\emptyset, \emptyset\rangle=\langle\emptyset, \emptyset\rangle$ and $\mathcal{R} c l\langle U, U\rangle=\langle U, U\rangle$.

3. $\operatorname{Rcl}(<\underline{R}(A), \bar{R}(A)>)$ is an $\mathcal{R}$-rough closed set. Hence, the result follows.

4. $\operatorname{Rcl}(<\underline{R}(A), \bar{R}(A)>\cup<\underline{R}(B), \bar{R}(B)>)=$ $\mathcal{R} c l(<\underline{R}(A) \cup \underline{R}(B), \bar{R}(A) \cap \bar{R}(B)>)$

$<\underline{R}(X), \bar{R}(X)>\supseteq \underline{D}<\underline{R}(A) \cup \underline{R}(B), \bar{R}(A) \cup \bar{R}(B)>$

$\Rightarrow \underline{R}(X) \supseteq \underline{R}(A) \cup \underline{R}(B), \bar{R}(X) \supseteq \bar{R}(A) \cup \bar{R}(B)$

$\Longrightarrow \underline{R}(X) \supseteq \underline{R}(A), \underline{R}(\bar{X}) \supseteq \underline{R}(B)$ and $\bar{R}(X) \supseteq \bar{R}(A), \bar{R}(X) \supseteq \bar{R}(B)$

$\Longrightarrow \underline{R}(X) \supseteq \underline{R}(A), \bar{R}(X) \supseteq \bar{R}(A)$ and $\underline{R}(X) \supseteq \underline{R}(B), \bar{R}(X) \supseteq \bar{R}(B)$

$\Rightarrow<\underline{R}(X), \overline{\bar{R}}(X)>\supseteq \underline{\underline{R}}(A), \bar{R}(A)>$ and

$$
<\underline{R}(X), \bar{R}(X)>\supseteq \geq \underline{R}(B), \bar{R}(B)>
$$

Thus, $\operatorname{Rcl}(<\underline{R}(A), \bar{R}(A)>\cup<\underline{R}(B), \bar{R}(B)>)$

$\supseteq \mathcal{R} c l(<\underline{R}(A), \bar{R}(A)>) \cup \operatorname{Rint}(<\underline{R}(B), \bar{R}(B)>)$. Again, $\operatorname{Rcl}(<\underline{R}(A), \bar{R}(A)>) \cup \mathcal{R} c l(<\underline{R}(B), \bar{R}(B)>)$ is an $\mathcal{R}$-rough closed set containing $\langle\underline{R}(A), \bar{R}(A)>$ and $<\underline{R}(B), \bar{R}(B)>$. Therefore,

$\mathcal{R} c l(<\underline{R}(A), \bar{R}(A)>) \cup \mathcal{R} c l(<\underline{R}(B), \bar{R}(B)>) \supseteq$ $\mathcal{R} c l(<\underline{R}(A), \bar{R}(A)>\cup<\underline{R}(B), \bar{R}(B)>)$.

So, $\operatorname{Rcl}(<\underline{R}(A), \bar{R}(A)>) \cup \mathcal{R} c l(<\underline{R}(B), \bar{R}(B)>)=$ $\mathcal{R} c l(<\underline{R}(A), \bar{R}(A)>\cup<\underline{R}(B), \bar{R}(B)>)$.

\section{Definition 4.3:}

Let $\langle\underline{\mathcal{R}}(\mathcal{T}), \overline{\mathcal{R}}(\mathcal{T})>$ be an $\mathcal{R}$-rough topology on $\mathrm{U}$. Then, the rough set $<\underline{R}(A), \bar{R}(A)>$ is said to be $\mathcal{R}$-dense, if $\operatorname{Rcl}(<\underline{R}(A), \bar{R}(A)>)=\langle U, U\rangle$.

\section{Theorem 4.5:}

Let $\langle\underline{\mathcal{R}}(\mathcal{T}), \overline{\mathcal{R}}(\mathcal{T})>$ be an $\mathcal{R}$-rough topology on $\mathrm{U}$. Then, a rough set $\langle\underline{R}(A), \bar{R}(A)\rangle$ is $\mathcal{R}$-dense iff every non-empty $\mathcal{R}$-rough open set has non-empty rough intersection with $<\underline{R}(A), \bar{R}(A)>$.

Proof:

First assume that $\langle\underline{R}(A), \bar{R}(A)\rangle$ is $\mathcal{R}$-dense. Then, $\mathcal{R} c l(\langle\underline{R}(A), \bar{R}(A)\rangle)=\langle U, U\rangle$. Consider a non-empty $\mathcal{R}$-rough open set $<\underline{R}(B), \bar{R}(B)>$. Then,

$<\underline{R}(A), \bar{R}(A)>\cap<\underline{R}(B), \bar{R}(B)>=\langle\emptyset, \emptyset\rangle$

$$
\begin{aligned}
& \Rightarrow \underline{R}(A) \cap \underline{R}(B)=\emptyset, \bar{R}(A) \cap \bar{R}(B)=\emptyset \\
& \Rightarrow \underline{R}(A) \cap \bar{R}(B)=\emptyset, \bar{R}(A) \cap \underline{R}(B)=\emptyset \\
& \Rightarrow \underline{R}(A) \subseteq(\bar{R}(B))^{C}, \bar{R}(A) \subseteq(\underline{R}(B))^{C} \\
& \left.\Rightarrow<\underline{R}(A), \bar{R}(A)>\subseteq<(\bar{R}(B))^{C}, \underline{R}(B)\right)^{C}> \\
& \Rightarrow<\underline{R}(A), \bar{R}(A)>\subseteq(<\underline{R}(B), \bar{R}(B)>)^{\mathrm{C}}
\end{aligned}
$$

Thus, $\quad(<\underline{R}(B), \bar{R}(B)>)^{\mathrm{C}}$ is an $\mathcal{R}$-rough closed set containing $<\underline{R}(A), \bar{R}(A)>$. Hence,

$\mathcal{R} c l(<\underline{R}(A), \overline{\bar{R}}(A)>) \subseteq(<\underline{R}(B), \bar{R}(B)>)^{\mathrm{C}}$.

Therefore, $\langle U, U\rangle \subseteq(<\underline{R}(B), \bar{R}(B)\rangle)^{\mathrm{C}}$. So, $\langle U, U\rangle$ $=(<\underline{R}(B), \bar{R}(B)\rangle)^{\mathrm{C}}$. Thus, $\langle\underline{R}(B), \bar{R}(B)\rangle=\langle\emptyset, \emptyset\rangle$. This is a contradiction. Therefore, $<\underline{R}(A), \bar{R}(A)>\cap<\underline{R}(B), \bar{R}(B)>=\langle\emptyset, \emptyset\rangle$.

Conversely, let every non-empty $\mathcal{R}$-rough open set has non-empty rough intersection with $\langle\underline{R}(A), \bar{R}(A)>$. Since, $\mathcal{R} c l<\underline{R}(A), \bar{R}(A)\rangle$ is an $\mathcal{R}$-closed set containing < $\underline{R}(A), \bar{R}(A)>,(\mathcal{R} c l<\underline{R}(A), \bar{R}(A)>)^{C}$ is an $\mathcal{R}$-open set which does not intersect $<\underline{R}(A), \bar{R}(A)>$. This is possible only when $(\mathcal{R} c l<\underline{R}(A), \overline{\bar{R}}(A)>)^{C}=\emptyset$. Then, $\mathcal{R} c l<$ $\underline{R}(A), \bar{R}(A)\rangle=\langle U, \bar{U}\rangle$. Therefore, $\langle\underline{R}(A), \bar{R}(A)\rangle$ is $\overline{\mathcal{R}}$-dense.

\section{CONCLUSION}

Though there are many studies on the topological properties of rough set approximations, only a few works have been done on the concept of rough topology. In this paper, an attempt has been made to define rough topology on an approximation space as a rough subset of the extended approximation space. The basic concepts of a topological 
space such as rough open set, rough closed set, rough interior and rough closure were extended to the proposed rough topological space and the properties were investigated.

\section{REFERENCES}

[1] M. E. Abd El-Monsef, O. A. Embaby and M. K. ElBably, "Comparison between Rough Set Approximations Based on Different Topologies", International Journal of Granular Computing, Rough Sets and Intelligent Systems, Vol. 3(4), 2014, pp $292-305$.

[2] E. A. Abo-Tabl, "Rough Sets and Topological Spaces Based on Similarity", International Journal of Mathematics Learning and Cybernetics, DOI 10.1007/s13042-012-0107-7.

[3] K. Anitha, "Rough Set Theory on Topological Spaces", Rough Sets and Knowledge Technology LNAI 8818, Springer 2014, pp 69 - 74.

[4] T. Herawan and M. M. Deris, "Rough Set Theory for Topological Spaces in Information Systems", in proceedings of Third Asia International Conference on Modelling and Simulation, 2009, pp 107 - 112.

[5] T. Herawan, "Roughness of Sets Involving Dependency of Attributes in Information Systems", International Journal Software Engineering and its Applications, Vol. 9(7), 2015, pp 111 - 126.

[6] M. J. Iqlan, M. S. Marouf and M. S. Shulaq, "Approximations of Sets in Topological Spaces", International Research Journal of Pure Algebra, Vol. 4(12), 2014, pp 636 - 643.

[7] T. B. Iwinski, "Algebraic Approach to Rough Sets", Bulletin of the Polish Academy of Science and Mathematics, Vol. 35, 1987, pp 673-683.

[8] J. Jarvinen, "The Ordered Structure of Rough Sets". in RSCTC 2004, LNAI 3066, eds S. Tsumoto et al., Springer- Verlag, Berlin, 2004, pp 49-58.

[9] J. Kelley, General Topology, Van Nostrand Company, 1955.

[10] M. Kondo and W. A. Dudek, "Topological Structures of Rough Sets Induced by Equivalence Relations", Journal of advanced computational Intelligence and Intelligent Informatics, Vol. 10(5), 2006 pp 621 - 624.

[11] M. Kondo, "On the Structure of Generalized Rough Sets", Information Sciences, Vol. 176, 2006, pp 589 -600 .

[12] E. F. Lashin, A. M. Kozae, A. A. Abo Khadra and T. Medhat, "Rough Set Theory for Topological Spaces", International Journal of Approximate Reasoning, Vol. 40, (2005, pp 35 - 43.

[13] Z. Li, "Topological Properties of Generalized Rough Sets", Proceedings of the Seventh International Conference on Fuzzy Systems and Knowledge, 2010, pp 2067 - 2070.

[14] Li, T. Xie and Q. Li, "Topological Structure of Generalized Rough Sets", Computers and Mathematics with Applications, Vol. 63, 2012, pp 1066 - 1071.
[15] W. J. Liu, "Topological Space Properties of Rough Sets", Proceedings of the third IEEE International Conference on Machine Learning and Cybernetics, Shanghai, 2004, pp 2353-2355.

[16] J. Mahanta and P. K. Das, "Topological Properties of Yao's Rough set", World Academy of Science, Engineering and Technology, Vol. 52, 2011, pp 922 $-924$.

[17] B. P. Mathew and S. J. John, "On Rough Topological Spaces", International Journal of Mathematical Archive, Vol. 3(9), 2012, pp 3413 3421.

[18] Z. Pawlak, "Rough Sets", International Journal of Computer and Information Sciences, Vol. 11(5), 1982, pp 341 - 356.

[19] Z. Pawlak, Rough Sets - Theoretical Aspect of Reasoning About Data, Kluwer Academic Publishers, The Netherlands, 1991.

[20] Z. Pei, D. Pei and L. Zheng, "Topology Vs Generalized Rough Sets", International Journal of Approximate Reasoning, Vol. 52, 2011, pp 231 239.

[21] Q. Qiao, "Topological Structure of Rough Sets in Reflexive and Transitive Relations", Proceedings of the Fifth International Conference on Bio-Medical Engineering and Informatics, 2012, pp 1585 - 1589.

[22] M. Ravindran and A. J. Divya, "A Study of Compactness and Connectedness in Rough Topological Spaces", International Journal of Mathematics, Vol. 12(6), 2016, pp 01 - 07.

[23] A. S. Salama, "Some Topological Properties of Rough Sets with Tools for Data Mining", International Journal of Computer Mathematics, Vol. 8(3)(2), 2011, pp 588 - 595.

[24] A. S. Salama and M.E. Abd El-Monsef, "New Topological Approach of Rough set Generalizations", International Journal of Computer Science Issues, Vol. 8(3)(2), 2011, pp 588 - 595.

[25] A. S. Salama, "Accurate Topological Measures for Rough Sets", International Journal of Advanced Research in Artificial Intelligence, Vol. 4(4), 2015, pp 31 - 37.

[26] M. L. Thivagar, C. Richard and N. R. Paul, "Mathematical Innovations of a Modern Topology in Medical Events", International Journal of Information Science, Vol. 2(4), 2012, pp 33 - 36.

[27] B. K. Tripathy and A. Mitra, "Some Topological Properties of Rough sets and their applications", International Journal of Granular Computing, Rough Sets and Intelligent Systems, Vol. 1(4), 2010, pp 355 - 369.

[28] Q. Wu, T. Wang, Y. X. Huang and J. S. Li, "Topology Theory on Rough Sets", IEEE Transactions on Systems, Man and Cybernetics part B. Cybernetics, Vol. 38(1), 2008, pp 68 -77.

[29] H. Yu and W. R. Zhan, "On the Topological Properties of Generalized Rough Sets", Information Sciences, Vol. 263, 2014, pp 141 - 152. 